\title{
VALORACIÓN SENSORIAL DE TOMATES VALENCIANOS DE PRODUCCIÓN ECOLÓGICA
}

\author{
M.D. Raigón, R. Monreal \\ Escuela Técnica Superior de Ingeniería Agronómica y del Medio Natural. \\ Universitat Politècnica de València. Camino de Vera, s/n. 46022 Valencia

\section{*mdraigon@gim.upv.es}

\section{Resumen}

La agricultura ecológica es un sistema productivo inocuo para el medio ambiente. Los sistemas de agricultura ecológica se basan en normas de producción concretas y precisas que tienen por objeto conseguir agroecosistemas que sean social y ecológicamente sostenibles. Se fundamenta en la reducción del uso de insumos externos, evitando el empleo de fertilizantes y plaguicidas sintéticos y potenciando el uso de variedades tradicionales. Contribuyendo a la revalorización de los saberes locales que suponen una herencia cultural considerable y a dar un paso hacia la recuperación de la autosuficiencia del agricultor en el uso de la semilla. A diferencia de las semillas comerciales, en la selección de las variedades locales han primado entre otros atributos, la calidad organoléptica, que los consumidores ecológicos valoran especialmente.

El objetivo del estudio es evaluar en siete variedades de tomate valenciano, cultivados bajo agricultura ecológica, los parámetros de acidez total, sólidos solubles, densidad, $\mathrm{pH}$ del jugo y el índice de color y de sabor del fruto. Por otro lado, se realiza una valoración organoléptica de los atributos de olor, color, textura y sabor. Se toman como referencia los mismos criterios en un tomate híbrido, de alta aceptación, de semilla certificada ecológica.

Existe una preferencia en cuanto a las tonalidades de tomates rojas y rosadas y hacia los calibres grandes. Los tomates mejor valorados por el olor han sido "Rosada de Castelló" y "Rossat Gran Xató". En cuanto al color los mejores valorados han sido las variedades de tomate de "Cuelga" y "Rosada de Castelló", respecto a la textura los tomates mejor valorados 
han sido la variedad "Masclet" y "Morado" y en cuanto al sabor las variedades más valoradas han sido "Rosada de Castelló" y "Masclet".

Palabras clave: variedades locales, organoléptico, físico-químico, índice de sabor, biodiversidad.

\section{Abstract}

Organic agricultura is a productive system which is harmless for the environment Organic agricultura systems are based on specific and precise production rules that have as an objective being socially and ecologically sustainable. It is based on the reduction of external inputs, avoiding the use of synthetic fertilizers and pesticides and favouring the use of traditional varieties. It also contributes to the enhancement of local knowledge, which represents a considerable cultural heritage, and to giving a step forward to the recovery of the self-sufficiency of farmers in the use of seed. Contrarily to commercial seed, in the selection of local varieties among other attributes has been prevailed organoleptic quality, which consumers of organic products especially value.

The aim of the study is evaluating in seven Valencian tomato varieties, grown under organic conditions, the total acidity, soluble solids, density, juice $\mathrm{pH}$ and colour and flavour indexes. On the other hand, and organoleptic assessment of the aroma, colour, texture and flavour attributes is performed. As a control a tomato hybrid, of high acceptance, of certified organic seed is used.

There has been a preference for tomatoes with red and pink tonalities and towards large sizes. The tomatoes best valued for the aroma have been "Rosada de Castelló" and "Rossat Gran Xató". Concerning colour the best valued ones have been the tomato varieties "Cuelga" and "Rosada de Castelló"; regarding texture the best valued tomatoes have been those of the varieties "Masclet" and "Morado" and regarding flavor the most valued varieties have been "Rosada de Castelló" and "Masclet".

Keywords: local varieties, organoleptic, physicochemical, flavour index, biodiversity. 


\section{1.- INTRODUCCIÓN}

Las variedades tradicionales surgen por el continuo esfuerzo de los agricultores en seleccionarlos materiales que respondan a las continuas necesidades del mercado. De este modo, las variedades locales han sido modeladas poco a poco en las sucesivas generaciones para responder a unas necesidades y exigencias de los consumidores (calidad organoléptica: textura, sabor, presencia, etc.), unos requerimientos productivos (resistencia a enfermedades y plagas, adaptación climática y edafológica, etc.) y unas premisas agroculturales (buena producción de semillas y fácil reproducción, adaptación a los sistemas de riego y fertilización tradicional, etc.) De este modo, siglos de selección natural y artificial han dado lugar a un patrimonio de una riqueza considerable en cuanto a ecotipos distintos, lugares y culturas diversas los han aprovechado.

La FAO define como variedades locales o tradicionales a "poblaciones diferenciadas, tanto geográfica como ecológicamente, que son visiblemente diferentes en su composición genética con las demás poblaciones y dentro de ellas, y que son producto de una selección por parte de los agricultores, resultado de los cambios para la adaptación, constantes experimentos e intercambios" (FAO, 1996; Hawtin et al., 1996; Domínguez et al., 1998).

El crecimiento del sector de los alimentos orgánicos supera con mucho al crecimiento de los sectores alimentarios convencionales, y los criterios en la elección de compra de los consumidores son la salubridad e inocuidad de los alimentos orgánicos, aunque la preocupación por la protección del medio ambiente, el incremento de la biodiversidad y el bienestar de los animales constituye también una motivación fundamental para esa elección (Garcia y Teixeira, 2017).

El tomate (Solanum lycopersicum L.) es una de las frutas más consumidas en el mundo, siendo el segundo cultivo vegetal más importante. Es un componente clave en la llamada dieta mediterránea, la cual está fuertemente asociada con la reducción de riesgo de enfermedades degenerativas y crónicas como son enfermedades del corazón o ciertos tipos de cáncer (Cruz et al., 2013). El tomate es una especie con alta variabilidad intraespecífica que conlleva diferentes formas de consumo, tanto en fresco, como triturado, frito y en un sin fin de conservas, pero cuya biodiversidad va desapareciendo del consumo, porque en el mercado se asientan las variedades comerciales e híbridas, homogéneas en cuanto a formas y 
composición. El objetivo de este estudio es evaluar parámetros físicoquímicos y la apreciación sensorial de una colección de tomates tradicionales de origen valenciano de producción ecológica, y compararlos con los valores obtenidos en una variedad híbrida de tomate de semilla certificada ecológica, que presenta una alta aceptación en el mercado.

\section{2.- MATERIAL Y MÉTODOS}

Los materiales vegetales estudiados en el presente trabajo han sido 7 variedades valencianas de tomate, conservadas por La Unió de Llauradors i Ramaders de Valencia, y una variedad comercial. Las siete variedades locales valencianas son "Masclet", "Pebre" o "Teticabra", "Cuarenteno", "Rosada de Castellón", "Morado", "Rossat Gran Xató" y de "Cuelga". Por otro lado, se incluyó en el estudio una variedad de tomate híbrido, de alta aceptación en el mercado, cuya semilla está clasificada como de procedencia ecológica (figura 1).

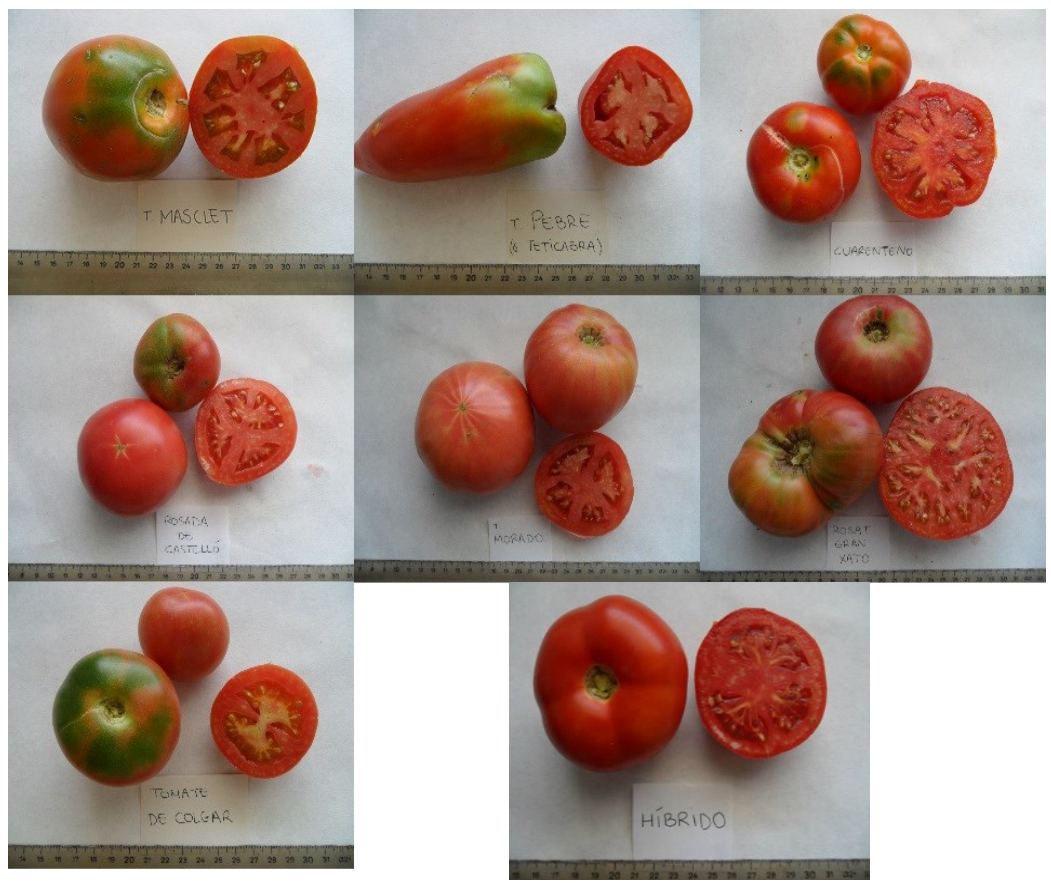

Figura 1.- Frutos de tomate de las variedades locales valencianas y de la variedad híbrida "Optima".

El cultivo de las diferentes variedades de tomate se realizó en las parcelas de agricultura ecológica certificada desde el año 2000, de La Unió 
de Llauradors i Ramaders en la Marjal del Moro de Sagunto (Valencia), mediante un diseño de bloques al azar con tres repeticiones por variedad. El terreno de se preparó con pase de fresadora simple y abonado de fondo en base de estiércol de oveja y cabra a razón de $1.5 \mathrm{~kg} / \mathrm{m}^{2}$. Seguidamente se efectuó un doble pase de fresadora dejando el suelo de cultivo preparado para la plantación. La plantación se realizó el 20 de abril de 2012. El riego fue a manta, por inundación de calles y con una frecuencia de una vez por semana. Las temperaturas medias del periodo de cultivo fueron de $15^{\circ} \mathrm{C}$ mínima y $30^{\circ} \mathrm{C}$ máxima. La recolección de los frutos de tomate comenzó a partir de mediados de junio y se extendió hasta finales de septiembre de 2012.

Con los frutos recolectados se procedió al análisis de los parámetros relacionados con las características del tomate, como el color, la densidad, el contenido en sólidos solubles, $\mathrm{pH}$, la acidez total valorable e índice de sabor.

El sistema Hunter empleado para la medida del color es un método objetivo que se basa en la medida de tres coordenadas: $\mathrm{L}=$ representa la luminosidad y varía de 0 a 100 (negro a blanco); $a=$ representa la variación de verde a rojo (-100 a 100); $b=$ representa la variación de azul a amarillo (100 a 100). El equipo empleado ha sido un espectrocolorímetro MINOLTA CR-300. Las medidas se realizan sobre los frutos de cada tratamiento. Y sobre cada fruto se llevan a cabo cinco medidas en puntos equidistantes del plano ecuatorial de la fruta. En cada medida se obtienen los valores de las tres coordenadas $(\mathrm{L}, \mathrm{a}, \mathrm{b})$ que combinadas entre sí dan lugar al índice de color. El índice de color (IC) de cada fruto se calcula mediante la fórmula:

$$
\text { I.C. }=\frac{1000 \times a}{L \times b}
$$

Con valores de índice de color próximos a 20, se evalúan las tonalidades rojas, a más índice de color más rojo, algo que se aprecia también en el zumo obtenido.

La determinación de la densidad del zumo de tomate se realiza por la relación entre la masa y el volumen del zumo del tomate homogeneizado.

La determinación del contenido en sólidos solubles presentes en el zumo de tomate se realiza mediante técnicas refractométricas (MAPA, 1994). Esta técnica puede ser utilizada porque el zumo es una disolución azucarada. El material utilizado en esta determinación es en un 
refractómetro de mano con un rango de $0-32{ }^{\circ}$ Brix. La medida se registra en ${ }^{\circ}$ Brix, que equivalen al contenido de sacarosa del zumo, en porcentaje $(\mathrm{m} / \mathrm{m})$ a $20^{\circ} \mathrm{C}$. Es decir, $1^{\circ}$ Brix $=1 \%$ (en peso) de sacarosa $\left(\right.$ a $\left.20^{\circ} \mathrm{C}\right)$. Si la temperatura del zumo es distinta a la de $20^{\circ} \mathrm{C}$ se corrige la medida obtenida en el refractómetro con la temperatura del zumo.

La determinación del $\mathrm{pH}$ se realiza por medida potenciométrica directa del zumo del tomate homogeneizado.

La determinación de la acidez total consiste en la valoración potenciométrica de la muestra con una disolución alcalina $(\mathrm{NaOH} 0.5 \mathrm{~N})$ hasta $\mathrm{pH}=8.1$ de la acidez del zumo, previa eliminación del dióxido de carbono de una muestra compuesta por la trituración y homogeneización de 3 tomates para cada variedad.

Para la medida se toman entre $20 \mathrm{~g}$ de la muestra exenta de $\mathrm{CO}_{2}$ diluida en $\mathrm{H}_{2} \mathrm{O}$ destilada, en un vaso de precipitados, se introduce el electrodo y se procede a la valoración con $\mathrm{NaOH} 0.5 \mathrm{~N}$ hasta el valor de $\mathrm{pH}=8$.1. Los resultados se expresan en gramos de ácido cítrico por cada 100 $\mathrm{g}$ de muestra.

$\mathrm{g}$ de ácido cítrico $/ 100 \mathrm{~g}=\frac{6.4 \cdot \mathrm{V} \cdot \mathrm{f} \cdot \mathrm{N}}{\mathrm{m}}$

Siendo, $\mathrm{N}=$ Normalidad del hidróxido de sodio, $\mathrm{V}=$ Volumen $(\mathrm{mL})$ de $\mathrm{NaOH} 0.5 \mathrm{~N}$, utilizados en la valoración, $\mathrm{m}=$ Peso (en g) de la muestra y $\mathrm{f}=$ Factor del hidróxido de sodio.

El índice de sabor del tomate (S) está basado en el contenido de azúcares y de ácidos del fruto (Navez et al.,1999) y se determina por la expresión: $\mathrm{S}=\mathrm{E}+\mathrm{I}$

Donde el índice de equilibrio (E) viene dado por la fórmula $E=\frac{10-\left(10-\frac{s}{a}\right)}{20}$ y el índice de intensidad (I) se calcula por la expresión $\mathrm{I}=\frac{\mathrm{a}}{10}$. En estas formulaciones $\mathrm{a}=$ concentración de ácidos, expresados en $\mathrm{g}$ de ácido cítrico por litro de muestra y $\mathrm{s}=$ concentración de azúcares totales (sólidos solubles), expresados en $\mathrm{g} \mathrm{L}^{-1}$.

Un valor de índice de sabor cercano a 1 está considerado arbitrariamente como ideal, acordándose por criterio una importancia equilibrada para los valores de $\mathrm{E}$ (índice de equilibrio) e I (índice de 
intensidad). Por otra parte, cuando el valor de $\mathrm{S}$ es inferior a 0.70 se considera que la variedad es mala respecto al sabor, y cuando el valor de $\mathrm{S}$ es superior a 0.85 se considera una variedad sabrosa.

La determinación de las características organolépticas del tomate, se realizó mediante una cata ciega, analizando los parámetros de calidad de una forma global y directa. La valoración se realiza según el test de Weiss (1981). Para la valoración, los tomates se cortan en el momento y no se les hace ningún tipo de tratamiento. Posteriormente se sitúan en platos, debidamente identificados por códigos y se procede a la valoración. Las características organolépticas determinadas son color global del tomate, textura, olor y sabor. Cada catador después de comprobar cada una de estas características, las calificará desde decadente hasta óptimo sobre la diagonal de una cuadrícula. Los datos obtenidos en la cuadrícula se extrapolan a un rango de 0 a 10 , donde el 0 es decadente y 10 es óptimo. Luego se realiza la media de los datos obtenidos por cada catador.

\section{3.- RESULTADOS Y DISCUSIÓN}

Los resultados promedios correspondientes a los parámetros analíticos de índice de color, densidad, contenido en sólidos solubles, $\mathrm{pH}$, acidez total valorable e índice de sabor en función del tipo de tomate se muestran en la tabla 1. La tabla 2 muestra los valores promedio de las apreciaciones de cata del color, olor, textura y sabor de los tomates. En todos los parámetros existe una alta variabilidad, debido a la diversidad de frutos. 
Tabla 1. Valor promedio del índice de color, densidad, sólidos solubles, $\mathrm{pH}$, acidez total e índice de sabor, en función del ecotipo del tomate

\begin{tabular}{|c|c|c|c|c|c|c|c|}
\hline Nombre & $\begin{array}{l}\text { Índice de } \\
\text { color }\end{array}$ & $\begin{array}{l}\text { Densidad } \\
(\mathrm{g} / \mathrm{mL})\end{array}$ & $\begin{array}{l}\text { Sólidos } \\
\text { solubles } \\
\left({ }^{\circ} \text { Brix) }\right.\end{array}$ & pH & $\begin{array}{c}\text { Acidez total } \\
\text { (\%) }\end{array}$ & $\begin{array}{c}\text { Sólidos } \\
\text { solubles } \\
\text { /acidez } \\
\text { total }\end{array}$ & $\begin{array}{c}\text { Índice } \\
\text { de } \\
\text { sabor }\end{array}$ \\
\hline Cuarenteno & $12.45 \pm 3.54$ & $0.974 \pm 0.01$ & $5.00 \pm 0.30$ & $4.27 \pm 0.11$ & $0.443 \pm 0.04$ & 11.31 & $0.997 \pm 0.03$ \\
\hline de Cuelga & $23.55 \pm 4.72$ & $0.950 \pm 0.01$ & $6.47 \pm 1.12$ & $4.15 \pm 0.03$ & $0.813 \pm 0.04$ & 7.91 & $1.169 \pm 0.08$ \\
\hline Masclet & $17.18 \pm 3.07$ & $0.980 \pm 0.01$ & $5.17 \pm 0.35$ & $4.33 \pm 0.03$ & $0.463 \pm 0.05$ & 11.20 & $1.013 \pm 0.03$ \\
\hline Morado & $28.61 \pm 4.13$ & $0.987 \pm 0.01$ & $5.30 \pm 0.56$ & $4.17 \pm 0.05$ & $0.543 \pm 0.14$ & 10.04 & $1.036 \pm 0.07$ \\
\hline Óptima & $28.65 \pm 3.21$ & $0.983 \pm 0.01$ & $7.63 \pm 0.25$ & $4.12 \pm 0.04$ & $0.677 \pm 0.07$ & 11.32 & $1.233 \pm 0.03$ \\
\hline $\begin{array}{l}\text { Pebre o } \\
\text { Teticabra }\end{array}$ & $13.04 \pm 3.74$ & $0.979 \pm 0.01$ & $5.00 \pm 0.72$ & $4.33 \pm 0.09$ & $0.433 \pm 0.03$ & 11.43 & $0.998 \pm 0.07$ \\
\hline $\begin{array}{c}\text { Rosada de } \\
\text { Castelló }\end{array}$ & $27.32 \pm 4.33$ & $0.969 \pm 0.02$ & $5.37 \pm 0.60$ & $4.15 \pm 0.14$ & $0.573 \pm 0.05$ & 9.36 & $1.023 \pm 0.06$ \\
\hline $\begin{array}{l}\text { Rossat Gran } \\
\text { Xato }\end{array}$ & $21.37 \pm 3.56$ & $0.979 \pm 0.03$ & $6.00 \pm 0.10$ & $4.42 \pm 0.05$ & $0.387 \pm 0.02$ & 15.54 & $1.156 \pm 0.01$ \\
\hline
\end{tabular}

Tabla 2. Valor promedio de los parámetros de cata del olor, color, textura y sabor, en función del ecotipo del tomate

\begin{tabular}{ccccc}
\hline Nombre & $\begin{array}{c}\text { Valoración } \\
\text { del olor }\end{array}$ & $\begin{array}{c}\text { Valoración } \\
\text { del color }\end{array}$ & $\begin{array}{c}\text { Valoración } \\
\text { de la textura }\end{array}$ & $\begin{array}{c}\text { Valoración } \\
\text { del sabor }\end{array}$ \\
\hline Cuarenteno & 5.07 & 6.16 & 4.52 & 4.51 \\
de Cuelga & 5.05 & 6.77 & 5.24 & 5.25 \\
Masclet & 6.47 & 5.12 & 5.86 & 5.79 \\
Morado & 6.25 & 6 & 5.82 & 5.24 \\
Óptima & 4.82 & 5.55 & 4.28 & 4.12 \\
Pebre o Teticabra & 5.02 & 3.83 & 5.75 & 5.25 \\
Rosada de Castelló & 6.70 & 6.37 & 5.59 & 6.19 \\
Rossat Gran Xato & 6.56 & 3.81 & 5.48 & 4.99 \\
\hline
\end{tabular}

Los índices de color en los tomates donde predominan las tonalidades rojas en la maduración presentan valores cercanos al 20, siendo 
inferiores en los tomates de las variedades donde existe presencia de tonalidades verdes en los hombros ("Cuarenteno" y "Masclet"). Los frutos con un valor superior al índice de color de 20, presentan tonalidades moradas, siendo el caso de los tomates rosados, típicos de la zona de Castellón ("Rosada de Castelló", con IC=27).

La densidad es un parámetro que se relaciona con la textura del fruto. En todos los casos, las densidades son inferiores a la unidad, por lo que se trata de frutos con mayor fracción volumétrica que gravimétrica, lo que proporciona frutos más jugosos en la degustación. El tomate híbrido es muy denso, siendo seguramente una de las características fijadas en la mejora. Lo superan los frutos de las variedades "Masclet" y "Morado".

Los azúcares presentes en el tomate son esencialmente la fructosa y la glucosa. La sacarosa está presente a un nivel muy escaso. Durante la fase de la maduración, cuando aparecen todos los pigmentos característicos del fruto, el contenido en azúcares aumenta y después se estabiliza, por ello, es tan importante que la recolección se realice lo más próxima posible al momento del consumo. La bibliografía indica que los contenidos en sólidos solubles presentes en tomates cultivados convencionalmente, fluctúan entre 5 y $8{ }^{\circ}$ Brix (Zambrano et al., 1996). De los resultados obtenidos se observa que la mayoría de las variedades presentan concentraciones de sólidos solubles superiores a $5{ }^{\circ} \mathrm{Brix}$. El nivel de sólidos solubles se relaciona con el sabor dulce, de manera que en este estudio la variedad híbrida (seguramente seleccionada para ello) es la que presenta los valores excepcionalmente altos de este parámetro. Aunque el contenido en sólidos solubles sea responsable de los sabores dulces, el sabor del tomate se aprecia por el conjunto entre la combinación de ácidos y azúcares.

Los valores de $\mathrm{pH}$ se encuentran dentro de lo indicado en bibliografía, siendo un parámetro poco variable dentro de la composición del tomate. No se encuentran diferencias estadísticas en los niveles del $\mathrm{pH}$ para las muestras analizadas, aunque el valor del $\mathrm{pH}$ del tomate híbrido es más bajo, lo que significa un tomate más ácido, confirmado por el valor de acidez total. Se observa la existencia de variedades muy ácidas como, el tomate de "Cuelga", posiblemente esta acidez, sea un parámetro que le permite la conservación natural.

El contenido de azúcares, ácidos y sus interacciones, determinan el sabor del tomate (Grierson y Kader, 1986) ya que según valores de $\mathrm{pH}$ inferiores a 4.4 y contenidos de sólidos solubles superiores a $4-4.5 \%$ son 
necesarios para un buen sabor, aunque varía según cultivares. Aunque el parámetro establecido para evaluar el sabor (independientemente de la cata), es el índice de sabor. Un valor de índice de sabor cercano a 1 está considerado arbitrariamente como ideal, acordándose por criterio una importancia equilibrada para los valores de E (índice de equilibrio) e I (índice de intensidad). En base a este criterio, el índice de sabor en los frutos de tomate analizados se puede considerar adecuado, siendo la variedad híbrida la que se aparta de este criterio Índice de sabor $=1.233$ ). Scott y Baldwin (1994) establecen que un valor alto de la relación sólidos solubles/acidez total se correlaciona con sabor suave, mientras que valores bajos se correlacionan con sabor ácido. Valores altos de la relación indican una excelente combinación entre los azúcares y los ácidos del tomate, en consecuencia mejor sabor. Según Kader et al. (1978) frutos de alta calidad contienen más de $0.32 \%$ de acidez total, $3 \%$ de sólidos solubles y la relación sólidos solubles/acidez total $>10$. En el presente trabajo se corrobora que los tomates mejor valorados superan el ratio de 10 en la relación sólidos solubles/acidez total.

En cuanto a la textura de los frutos se observa que el tomate peor valorado ha sido el de la variedad híbrida (4.28), para el resto de los tomates, la valoración está próxima al valor de 5 (sobre 10). Respecto al sabor obtenido en la valoración sensorial se observa que las variedades de tomate mejor valoradas son las de mayor calibre, tipo ensalada. Los frutos peor valorados respecto a su sabor son los de la variedad híbrida, con diferencias significativas frente al resto de las variedades (4.12), frente al mejor valorado (6.19) del tomate "Rosada de Castellón". Variedad de tomate que se caracteriza también por la mejor valoración en cuanto al aroma que desprenden sus frutos y el color que los catadores aprecia.

La figura 2 muestra la diferencia, en cada uno de los cuatro atributos sensoriales, de las puntuaciones obtenidas por los tomates de las variedades valencianas frente a la nota de cata obtenida por los frutos de la variedad híbrida. Se observa que en todos los casos el sabor está valorado por encima del tomate híbrido, que la textura excepto en los frutos de las variedades "Cuarenteno" y de "Cuelga" que son frutos más duros para aguantar la conservación natural, también está mejor valorada en las variedades locales. El olor, excepto en los tomates de la variedad de "Cuelga", que son frutos poco aromáticos, se valora para el resto de variedades, por encima del tomate híbrido de referencia. Para el atributo del color, las valoraciones que superan al tomate híbrido son las de tonalidades rosadas y moradas, y no se 
valoran positivamente los tomates con pigmentaciones verdes en los hombros.

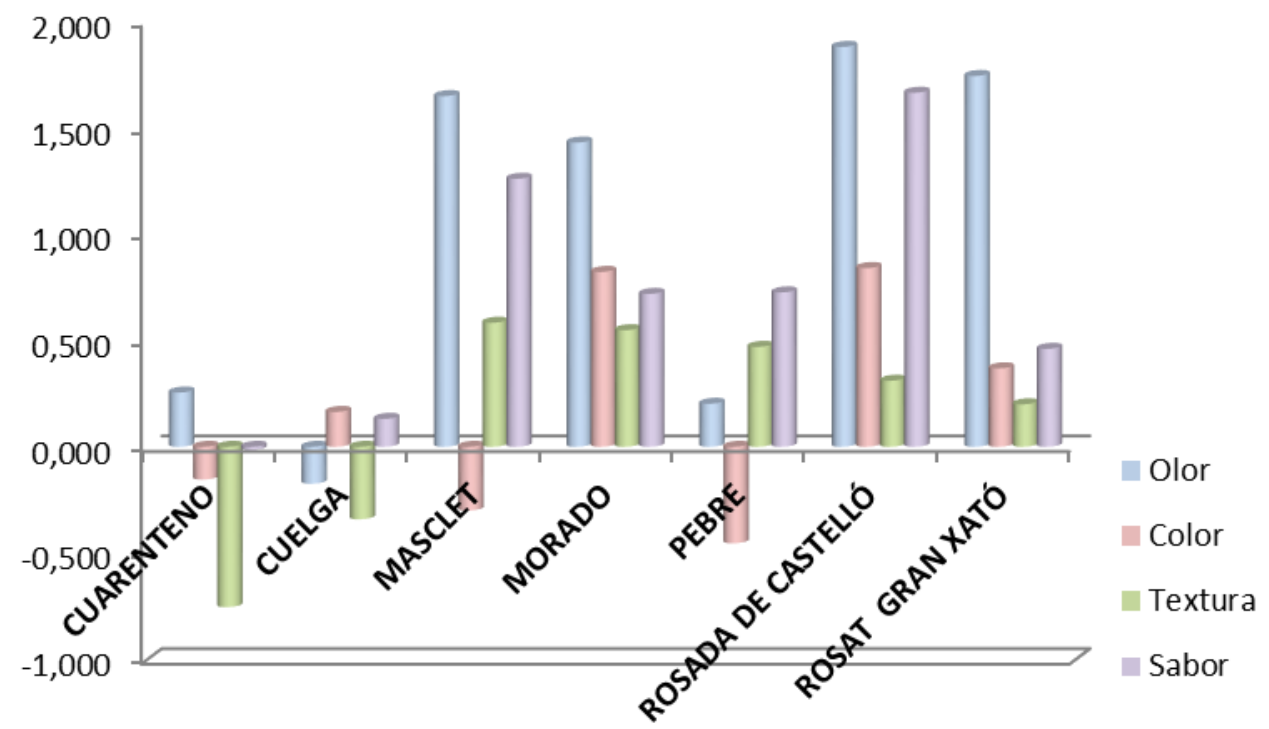

Figura 2.- Valoración de distintas características orgalonépticas de las variedades locales valencianas y de la variedad híbrida "Optima".

\section{4.- CONCLUSIONES}

Las variedades de tomates valencianas con tonalidades rosadas y calibres grandes, como "Morado", "Rosada de Castelló" y "Rossat Gran Xató" son los mejor valorados. Los tomates de variedades tradicionalmente valencianas, cultivados en cultivo ecológico, presentan muy buena valoración organoléptica, siendo un argumento de peso para recuperar su posicionamiento en el mercado frente a las variedades híbridas.

\section{REFERENCIAS BIBLIOGRÁFICAS}

Cruz, R.M.; González, J.; Sánchez, P. 2013. Propiedades funcionales y beneficios para la salud del licopeno. Nutrición Hospitalaria 28: 6-15.

Domínguez, A.; Roselló, J.; Rodrigo, M.I. 1998. Tipificación y estudio productivo de diversas variedades tradicionales de tomate, calabaza y melón cultivados con métodos ecológicos. En: Libro de resúmenes del III 
congreso de la Sociedad Española de Agricultura Ecológica: una Alternativa para el Mundo Rural del Tercer Milenio. Valencia. 315-322 pp.

FAO, Food and agriculture Organization of the United Nations. 1996. The State of the World's Plant Genetic Resources for Food and Agriculture. FAO, Roma, Italia.

Garcia, J.M.; Teixeira, P. 2017. Organic versus conventional food: A comparison regarding food safety. Food Reviews International 33(4), 424446.

Grierson, D.; Kader, A.A. 1986. Fruit ripening and quality. In J. G. Atherton \& J. Rudich (Eds.) The tomato crop: A scientific basis for improvement (pp. 241-280). London: Chapman and Hall.

Hawtin, G.C.; Iwanaga, M.; Hodgkin, T. 1996. Genetic resources in breeding for adaption. Euphytica 92: 255-266.

Kader, A.A.; Morris, L.L.; Stevens, M.A.; Albright-Holton, M. 1978. Composition and flavor quality of fresh market tomatoes as influenced by some postharvest handling procedures. J. Amer. Soc. Hort. Sci. 103(1): 6-11.

MAPA. Ministerio de agricultura pesca y alimentación. 1994. Métodos oficiales de análisis. Tomo II. Madrid. 567 pp.

Navez, B.; Letard, M.; Graselly, D.; Jost, M. 1999. Les critéres de qualité de la tomate. Infos-Ctifl. 155: 41-47.

Scott, J.W.; Baldwin. E.A. 1994. Effect of harvest stage on solids, acids, firmness and shelf life of tomato. Hort. Science 29(5): 566.

Weiss, D.S. 1981. The impossible dream of Fechner and Stevens. Perception 10: 431-434.

Zambrano, J.; Moyeja, J.; Pacheco, L. 1996. Efecto del estado de madurez en la composición y calidad de frutos de tomate. Agronomia Tropical 46(1): 61-72. 